\title{
SPECTROSCOPY OF CHARGED DONORS AND MANY-ELECTRON EFFECTS IN SEMICONDUCTOR QUANTUM WELLS
}

\author{
B.D. McCombe, Z.X. Jiang, J.G. Tischler, B.A. Weinstein
}

Department of Physics and Center for Advanced Photonic and Electronic Materials SUNY at Buffalo, Buffalo, NY 14260, USA

AND P. HAWRYLAK

Institute for Microstructural Sciences, NRC, Ottawa, Canada K1A OR6

Far infrared magnetospectroscopic studies of negative donor ions $\left(\mathrm{D}^{-}\right)$, and donors in the presence of many excess electrons in high magnetic fields in GaAs/AlGaAs quantum wells are reviewed. Both singlet and triplet transitions of well-center $\mathrm{D}^{-}$-ions were observed and are in good agreement with recent theoretical calculations. For off-well-center $\mathrm{D}^{-}$-ions evidence for a predicted magnetic-field-induced "unbinding" of the second electron was found. In the presence of many excess electrons the $\mathrm{D}^{-}$-singlet and -triplet transitions are blue-shifted substantially and evolve into bound magnetoplasmon excitations. Cusps are observed at integral and fractional Landau-level filling factors $(\nu)$ in a plot of normalized blue-shift of the $\mathrm{D}^{-}$-singlet-like bound magnetoplasmon transition vs. $\nu$. For $\nu<1$, the singlet-like bound magnetoplasmon transition continuously approaches the isolated $\mathrm{D}^{-}$-singlet transition with increasing magnetic field, while the triplet-like transition loses strength, irrespective of the electron density. Exact diagonalization studies of a donor ion with a few electrons in a parabolic lateral confining potential show the importance of electron-electron interactions and localization due to the long-range fluctuating potential in explaining this behavior. High pressure studies in a specially designed diamond anvil cell exhibit a continuous evolution from bound magnetoplasmon transitions to isolated $\mathrm{D}^{-}$-transitions to neutral donor transitions in a single sample as the pressure is increased and the electron density in the wells is decreased.

PACS numbers: 73.20.Dx, 73.20.Hb, 78.66.Fd

\section{Introduction}

With modern growth techniques very thin semiconductor layers can be produced with monolayer precision, selectively doped with impurities in well-defined regions. Placement of impurities in the growth direction can be controlled to within 
a few atomic layers. This has allowed detailed experimental investigations of the effects of confinement and impurity placement on shallow neutral donor $\left(\mathrm{D}^{0}\right)$ and acceptor states by various spectroscopic means, principally near IR photoluminescence (PL) spectroscopy and far infrared transmission or photoconductivity spectroscopies. A combination of experimental and theoretical studies has led to a good understanding of the electronic states of neutral donors (and to a lesser degree acceptors) in quasi-two-dimensional semiconductor structures: the binding and transition energies increase with increasing confinement and with increasing magnetic field, and decrease as the donor/acceptor ion is moved away from the on-well-center position [1].

Experimental and theoretical efforts over the past eight to nine years have focused on identification of stable negative donor ions $\left(\mathrm{D}^{-}\right)$in quantum-well structures [2] and on understanding the various two-electron energy states in high magnetic fields $[3,4]$. Since the correct identification of the $\mathrm{D}^{-}$-singlet transition via far infrared (FIR) magnetospectroscopy [2], additional studies have verified and extended these results [3], and the nature of the singlet states and their field- and well-width dependencies are well understood for on-well-center $\mathrm{D}^{-}$ions. The $\mathrm{D}^{-}$-triplet transitions have not been as extensively investigated, and there is disagreement in the literature between theory and experiment [5-7]. However, as discussed below, recent improvements in the calculations have now removed this discrepancy, and agreement is very good. For off-well-center $\mathrm{D}^{-}$-ions, there are theoretical predictions of a magnetic-field-induced unbinding of the ground state [8-10], and far infrared spectroscopic measurements have verified some of these predictions [11]. Far infrared studies of a series of samples in which the electron density in the wells has been varied from "zero" to values much larger than the donor density have revealed many-body effects at high fields when the Landau level filling factors $(\nu)$ lie in the range $2 \geq \nu \geq 0.1$ [12-14].

In the next sections we present a short summary of the essential theory, followed by a description of far infrared studies of negatively charged donors in quantum wells, both on-center and off-center. This is followed by a discussion of investigations of a series of $\mathrm{GaAs} / \mathrm{Al}_{0.3} \mathrm{Ga}_{0.7} \mathrm{As}$ multiple-quantum-well (MQW) samples with Si donors $\delta$-doped both in the well-centers and in the wide barrier-centers which, in concert with a theoretical model, reveal interesting many-electron and localization effects [4]. Finally, some recent experiments which employ high pressures to tune the electron density in individual samples are described.

\section{Theoretical background}

In the one-band, effective-mass approximation the Hamiltonian for two electrons in a quantum well of height $V_{0}$ and width $L$ with a positive donor ion located at the center of the well (the confined $\mathrm{D}^{-}$-ion) can be written as

$$
H=\sum_{j=1}^{2}\left\{\frac{1}{2 m_{\mathrm{e}}^{*}}\left[p_{\mathrm{e} j}+\frac{e \boldsymbol{A}\left(r_{\mathrm{e} j}\right)}{c}\right]^{2}+V\left(z_{\mathrm{e} j}\right)\right\}-\frac{e^{2}}{\varepsilon r_{\mathrm{e} 1}}-\frac{e^{2}}{\varepsilon r_{\mathrm{e} 2}}+\frac{e^{2}}{\varepsilon\left|r_{1}-r_{2}\right|}
$$

where $V\left(z_{j}\right)=V_{0}\left(\left|z_{j}\right|>L / 2\right)$ and $V\left(z_{j}\right)=0\left(\left|z_{j}\right|<L / 2\right), r_{\mathrm{e} j}$ is a vector in the plane of the quantum wells specifying the position of electron $j$, and $\dot{A\left(r_{e j}\right)}$ is the vector potential of a uniform external magnetic field in the $z$-direction. 
Equation (1) describes the one-electron case, $\mathrm{D}^{0}$, with the last two terms of Eq. (1) set equal to zero and only one term kept in the sum. For this case a variational approach has often been employed to obtain solutions for the allowed energy levels [15].

It has been common practice in the literature to label the quasi-two-dimensional (2D) states of shallow neutral donor impurities over a wide range of magnetic fields by the usual three-dimensional set of hydrogenic quantum numbers $\left(n, l, M_{z}\right)$ appropriate to low magnetic fields; here $n$ is the principal quantum number, $l$ - the total orbital angular momentum quantum number, and $M_{z}$ is the quantum number for projection of angular momentum. This is imprecise at best, unless the use is clarified by establishing a clear correspondence between the 3D states and the (proper) quasi-2D states [16]. We will continue to follow this convention with the $3 \mathrm{D}$ quantum numbers used in the following sense: the $3 \mathrm{D}$ hydrogenic states $\left(n, l, M_{z}\right)$ used to label the quasi-2D states are those to which quasi-2D, high-field states track continuously as the field is reduced and the quantum-well width approaches infinity.

In the very high magnetic field, $2 \mathrm{D}$ limit with $\boldsymbol{B} \perp 2 \mathrm{D}(x-y)$ plane, appropriate quantum numbers for this problem are the orbit center quantum number, $O$ (corresponding to eigenvalues of the operator for the square of the radius of the cyclotron orbit center, $r_{0}^{2}$ ), and $N$, the Landau quantum number (corresponding to the eigenvalues of the operator for the radius of the cyclotron orbit about the center). The eigenvalues and eigenfunctions of these operators provide the exact solution for free-electron motion in a uniform external magnetic field. For each Landau level (LL) one allowed state corresponds to the area between adjacent circles of radius $r_{0}, A=2 \pi \ell^{2}$, where $\ell=(\hbar e / c B)^{1 / 2}$. The LL degeneracy $\eta=1 / 2 \pi \ell^{2}$, and the filling factor $\nu=n_{s} / \eta$. The operator for the $z$-component of angular momentum, $L_{z}$ (eigenvalues $\hbar M_{z}=0, \pm \hbar,+2 \hbar, \ldots$ with $M_{z} \leq N$ ), commutes with the full Hamiltonian at all values of $B$ for arbitrary confinement with the magnetic field aligned along the confinement direction. In the infinite field limit $M_{z}=N-O$. The quantity $\left|M_{z}\right|$ is proportional to the mean distance of the electron from the origin and is thus useful in understanding physically how a positively charged impurity ion at the origin perturbs the LLs.

The $D^{0}$ states and allowed transitions associated with the two lowest LLs are shown in Fig. 1a for a high-field situation. The parity of the wave functions is determined by $N+O,(-1)^{N+O}$, and since $M_{z}=N+O-2 O,(-1)^{N+O}=$ $(-1)^{M_{z}}$. Therefore, the electric-dipole selection rules are determined completely by $\Delta M_{z}= \pm 1$ (and $\Delta O=0$ in the infinite field limit).

For the two-electron $\left(\mathrm{D}^{-}\right)$case Figs. 1b (singlet) and 1c (triplet) show schematically the $\mathrm{D}^{-}$energy levels (energy of the second electron) and transitions of interest in the high magnetic-field limit for a 2D system. Solutions for this problem have been obtained by MacDonald [4]. The total orbit center quantum number $O=O_{1}+O_{2}$ and total Landau quantum number $N=N_{1}+N_{2}$ label the states in this case. Dzyubenko and Sivachenko [4] have obtained numerical solutions for the $\mathrm{D}^{-}$-ion confined in finite quantum wells; the dominant electric-dipole transitions are indicated in Figs. $1 \mathrm{~b}$ and $\mathrm{c}$. In this case the selection rules are governed by $\Delta M=\Delta\left(M_{z 1}+M_{z 2}\right)=+1\left(\right.$ and $\Delta O=\Delta\left(O_{1}+O_{2}\right)=0$ in the infinite 
(a)

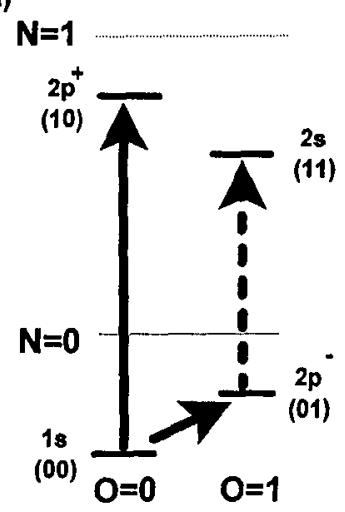

(b)

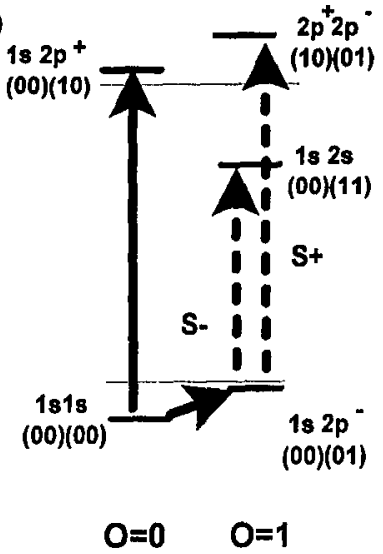

(c)

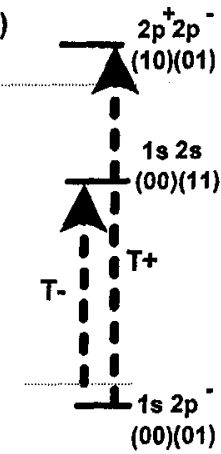

Fig. 1. Schematic diagrams of energy states associated with the two lowest LLs for (a) one electron $\left(\mathrm{D}^{0}\right)$, and the second electron for the $\mathrm{D}^{-}$-singlet (b) and -triplet (c) states. Allowed transitions are shown by arrows; the dashed arrows indicate transitions that originate in an excited state. The $\mathrm{D}^{-}$-states are labeled by individual one-electron $\mathrm{D}^{0}$ states in both the $2 \mathrm{D}$, high-field notation $(N, O)$ and the low-field, 3D hydrogenic notation $\left(n, l, M_{z}\right)$.

field limit). The dominant transitions at high fields are vertical in these diagrams with the singlet and $\mathrm{T}^{-}$-triplet transitions having the largest oscillator strengths.

The basis for the ground singlet-state is the symmetric orbital combination of $(00)(00)$ and the final state is the symmetric orbital combination of $(00)(10)$. Since the Coulomb repulsion for the two electrons plays a strong role, and this positive energy is larger for the symmetric $(00)(00)$ ground state than for the $(00)(10)$ excited state (the (10) orbital is more extended), the singlet transition energy is red-shifted from its single-particle "parent". The parent single-particle transition in this case is the $\mathrm{D}^{0}(00)-(10), 1 s-2 p^{+}$in the low-field, 3D notation.

The parent single-particle transition for the $\mathrm{T}^{-}$-triplet transition is the $\mathrm{D}^{0}(01)-(11), 2 p-2 s$ in the low-field, 3D notation. This transition lies below cyclotron resonance (CR) because the (11) final state corresponds to an electron with maximum probability density at the position of the positive donor ion. This configuration leads to a larger binding energy of the (11) state with respect to the $N=1 \mathrm{LL}$ than the corresponding binding energy of the (01) state with respect to the $N=1 \mathrm{LL}$. Simple qualitative considerations similar to those above would suggest that the $\mathrm{T}^{-}$-transition should be blue-shifted (by a small amount due to the antisymmetric orbital combination) from its single-particle $\left(\mathrm{D}^{0}\right)$ parent. The more extended single-particle orbital of one electron in the initial state (01) combined with the more compact single-particle orbital of one electron in the final state (11) could lead to a larger binding of the second electron in the final state than in the initial state. However, these simple arguments must be modified by the change in average negative Coulomb energy of attraction of each electron to the positive donor ion due to the actual two-electron wave function, and proper numerical calculations show that this state is actually very slightly red-shifted from its parent single-particle counterpart. To observe the triplet transitions it is nec- 

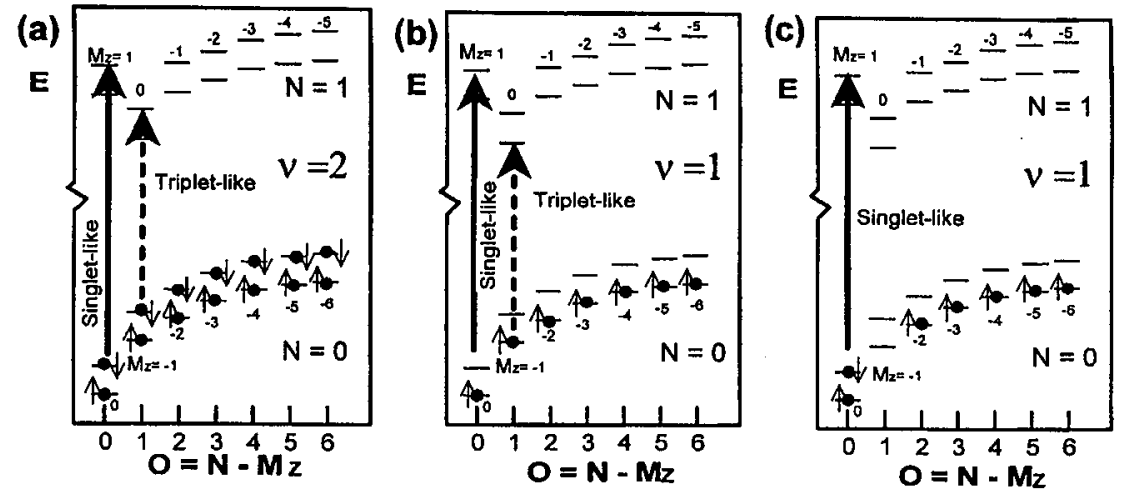

Fig. 2. Schematic diagrams of single-particle energies of states $|N, O\rangle$, in the $N=0$ and $N=1$ LLs in the high-field limit for (a) a completely filled LL $(\nu=2)$, (b) a spin-polarized (SP) state $(\nu=1)$, and (c) a spin-wave $(\mathrm{SW})$ state $(\nu=1)$. Filled circles represent electrons. The arrows indicate the electric-dipole-active transitions (after Hawrylak [17]).

essary to populate the triplet ground state $(00)(01)$ thermally, since it lies above the singlet ground state over the field range of interest.

For a positive donor ion in the presence of a large density of electrons the repulsive interaction among all electrons, as well as the attractive interaction between each electron and the positive ion, must be taken into account. It is helpful to consider the single-particle energy states of an electron in the presence of a positive donor ion in the high-field, 2D limit (Fig. 2) to understand some basic features of this complex situation. Numerical solutions of the appropriate Hamiltonian in the high-field limit at $\nu=2$ and 1 have been obtained independently by two groups [17]. Both predict a dominant transition from the ground state that has evolved from the two-electron singlet state (a singlet-like transition) and which is blue-shifted (predominantly due to exchange) by an amount that depends on the field and electron density. A blue-shifted triplet-like transition from a populated, higher energy state of the system is also predicted. These collective excitations, the bound magnetoplasmons, retain much of the character of the respective two-electron transitions, due to the strong attraction of the positive donor ion to electrons in its immediate vicinity. The population of the single-particle states that make up the collective ground and excited states associated with the $N=0 \mathrm{LL}$ is indicated in Figs. $2 a$ and $b$ for $\nu=2$ and 1 , respectively. In the latter case a spin-polarized ground state is assumed. In this situation the singlet-like transition should vanish at the lowest temperatures, and only the triplet-like transition should remain. Hawrylak [17] has shown that for small enough spin splitting, the ground state of the many-electron system is not spin polarized at $\nu=1$. Rather, it is a "spin-wave" state, approximately a singlet two-electron complex localized at the position of the donor ion and a partial hole in the state with $O=1$, with additional oscillations in spin as $O$ becomes larger. This is shown schematically in Fig. 2c. Since the triplet-like transition requires population of the $O=1$ state, a very weak triplet-like transition at low temperatures, and a strong singlet-like 
transition are expected for the spin-wave state, in contrast to the spin-polarized ground state for which the singlet-like bound magnetoplasmon transition should vanish at zero temperature.

\section{Results}

\subsection{The $T^{-}$-triplet transition}

As mentioned above, in addition to the well-understood dominant transitions involving singlet final states associated with each of the lowest two LLs (see Fig. 1b), two triplet transitions have been predicted by Dzyubenko and Sivachenko [4]. One of these, $\mathrm{T}^{-}$, lies slightly below CR (its parent is the $\mathrm{D}^{0} 2 p^{-}-2 s$ transition, Fig. 1c) and one, $\mathrm{T}^{+}$, lies at higher energies between the dominant singlet line (its parent is $\mathrm{D}^{0} 1 s-2 p^{+}$transition, Fig. 1c). The calculated position of the singlet line agrees well with experiments. A weak feature observed about $13 \mathrm{~cm}^{-1}$ below $C R$ was attributed to the $\mathrm{T}^{-}$-triplet transition in early work [5], but a later experimental and theoretical investigation [6] contested this assignment. A calculation [6] predicted the $\mathrm{T}^{-}$-line to lie only about $1 \mathrm{~cm}^{-1}$ below $\mathrm{CR}$, too close to be resolved. In these experiments an observed feature about $10 \mathrm{~cm}^{-1}$ below $\mathrm{CR}$ was attributed to the $\mathrm{D}^{0} 2 p^{-}-2 s$ transition. Later FIR laser and Fourier transform infrared (FTIR) magnetospectroscopic studies of the temperature dependence of various transitions in several samples [7] showed two lines below $\mathrm{CR}$. These were attributed to the $\mathrm{D}^{0} 2 p^{-}-2 s$ transition $\left(9 \mathrm{~cm}^{-1}\right.$ below $\left.\mathrm{CR}\right)$ and the $\mathrm{T}^{-}$-triplet line $\left(13 \mathrm{~cm}^{-1}\right.$ below $\left.\mathrm{CR}\right)$.

Examples of laser magnetophotoconductivity results are shown in Fig. 3. The upper trace shows photoresponse for a sample doped in the well centers at $2 \times 10^{10} \mathrm{~cm}^{-2}$. The $\mathrm{D}^{0} 1 s-2 p^{+}$transition, the $\mathrm{D}^{-}$-singlet, $\mathrm{CR}$, the feature attributed to the $\mathrm{D}^{0} 2 p^{-}-2 s$ transition, and the feature attributed to the $\mathrm{T}^{-}$-triplet transition are all clearly seen, as is a broad feature that may be related to $\mathrm{T}^{+}$. The middle trace shows similar spectra for a sample with the same well doping but also doped in the centers of the $60 \mathrm{~nm}$ barriers at $3.5 \times 10^{10} \mathrm{~cm}^{-2}$. In this case the feature attributed to the $\mathrm{T}^{-}$-transition is much stronger. For a more heavily barrier-doped sample (lowest trace in Fig. 3) with the same well doping, the $\mathrm{D}^{0}$ line is very weak with the $\mathrm{D}^{-}$-singlet transition dominating the spectrum. The weak feature at about $6 \mathrm{~T}$ may be due to a predicted singlet transition from the ground $(O=0)$ state to an excited state associated with $N=1$ having $O=2$. The only surviving feature at fields above CR (energies below $\mathrm{CR}$ ) is that occurring at the highest field (attributed to the $\mathrm{T}^{-}$-triplet). This systematic behavior is consistent with the assignment of this highest field feature to a $\mathrm{D}^{-}$-related transition. A comparison of the detailed temperature dependencies of the various lines [7] in several samples with the same well doping but varying barrier doping is also consistent with the assignment of this feature to an excited state of $\mathrm{D}^{-}$. The $\mathrm{T}^{-}$-transition appears to be the most likely origin of this line, and improved calculations for this structure now predict the $\mathrm{T}^{-}$-line to occur very close to the experimental observations, namely red-shifted by about $3 \mathrm{~cm}^{-1}$ from the parent $\mathrm{D}^{0}$ transition [18]. Some recent FTIR results over a wide range of magnetic field at a temperature of $20 \mathrm{~K}$ obtained on another section of the sample of the middle trace of Fig. 3 are 


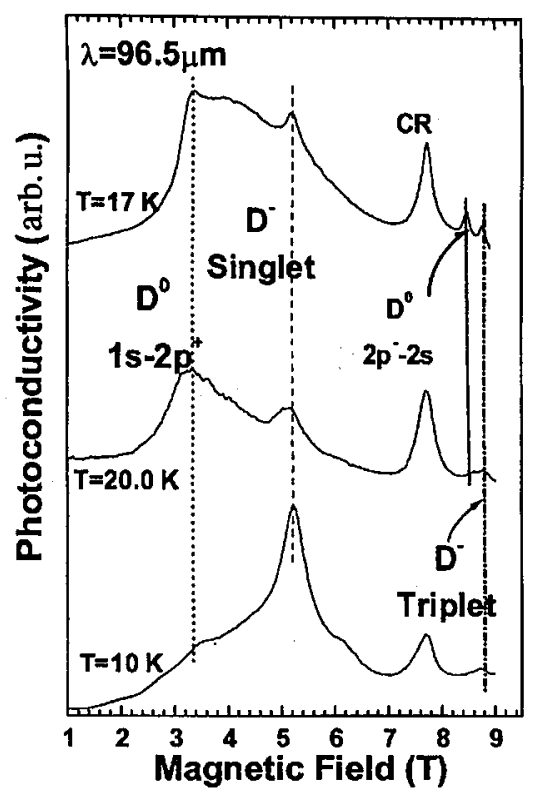

Fig. 3. FIR photoconductivity scans with the $96.5 \mu \mathrm{m}$ laser line for three well-center $\delta$-doped (all at $2 \times 10^{10} \mathrm{~cm}^{-2}$ ) multiple-quantum-well samples (well width $20 \mathrm{~nm}$ ): upper - no barrier doping; middle - barrier centers $\delta$-doped at $3.5 \times 10^{10} \mathrm{~cm}^{-2}$; and lower - barrier centers $\delta$-doped at $4 \times 10^{10} \mathrm{~cm}^{-2}$.

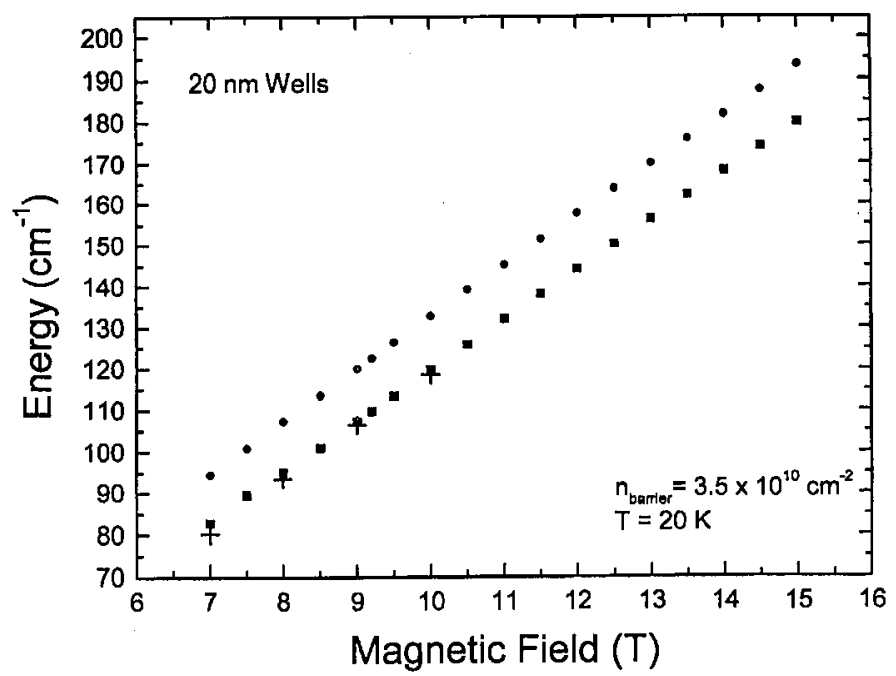

Fig. 4. Plot of the frequency of the $\mathrm{T}^{-}$-triplet feature ( $\square$ ) vs. magnetic field. The electron CR positions $(\bullet)$ are shown for reference. Calculated results for the $\mathrm{T}^{-}$-triplet transition are shown at several fields by the +'s (after [18]).

shown in Fig. 4. For comparison, recently calculated values of the $\mathrm{T}^{-}$-transition energy [18] are shown by the crosses at several fields. Agreement is excellent. 


\subsection{Off-well-center $D^{-}$-ions}

Predictions of interesting magnetic-field behavior for off-well-center doped $\mathrm{D}^{-}$-ions, including singlet-triplet crossover and magnetic-field-induced unbinding of electrons led to an experimental FIR magnetospectroscopy study of barrier-center and off-well-center doped samples [11], and a subsequent detailed theoretical study for the experimental situation [19].

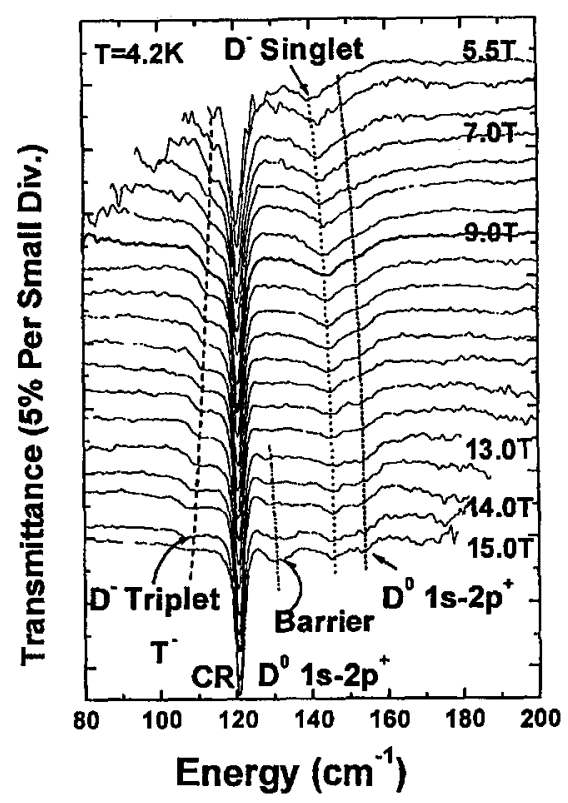

Fig. 5. Magnetotransmission spectra for the off-well-center-doped sample (well-width $20 \mathrm{~nm}$ ) at $4.2 \mathrm{~K}$ at magnetic fields between $5.5 \mathrm{~T}$ and $15 \mathrm{~T}$ in steps of $0.5 \mathrm{~T}$. CR lines for spectra at different fields are aligned to the CR position of the $9 \mathrm{~T}$ spectrum to show clearly the behavior of the other features.

An example of magnetotransmission data for a $20 \mathrm{~nm}$ well-width MQW sample $\delta$-doped in the $(60 \mathrm{~nm})$ barrier-centers at $3 \times 10^{10} \mathrm{~cm}^{-2}$ and in the (20) wells at a position 3/4 of the way to the edge is shown in Fig. 5 . Several features are noteworthy. First, the strength of the off-well-center $\mathrm{D}^{-}$-singlet line decreases continuously as field is increased from 5.5 to $15 \mathrm{~T}$. Second, an off-well-center $\mathrm{D}^{0} 1 s-2 p^{+}$transition appears at high fields, concomitant with the decrease in the $\mathrm{D}^{-}$feature. Third, an off-well-center $\mathrm{T}^{-}$-triplet line observed initially about $7-8 \mathrm{~cm}^{-1}$ below $\mathrm{CR}$ shifts to lower frequency relative to $\mathrm{CR}$, gains strength initially, and finally loses strength at the highest fields. The weak feature about 10-12 $\mathrm{cm}^{-1}$ above CR appearing at fields above $13 \mathrm{~T}$ is apparently due to accidental neutral donors in the barriers near the edge.

These features and their behavior can be understood qualitatively from simple physical arguments which agree with detailed calculations $[8,18]$. The binding of the "second" electron depends sensitively on competition between the attrac- 
tive potential of the position donor ion and the repulsive potential of the other electron. As the positive ion is moved away from the well center, the maximum in the probability density along the quantum well direction remains very close to the well center; thus the attractive potential energy decreases, while the repulsive potential energy and the kinetic energy are relatively unchanged. Hence the binding energy is expected to decrease rapidly as the donor ion is moved away from the well center. An external magnetic field shrinks the wave functions of the electrons in the plane normal to the field direction, increasing the repulsive potential energy substantially, while the attractive potential energy of each electron to the ion is increased only slightly. This effect is strongest for the spin-singlet ground state. The lowest spin-triplet antisymmetric orbital combination involves a more extended basis orbital and also tends to keep the electrons farther apart on average. As the field increases, the singlet-ground state binding energy decreases faster than the triplet ground state; they become comparable at fields near $10 \mathrm{~T}$. Thus at high fields and at $4.2 \mathrm{~K}$, both the singlet and triplet states are easily depopulated, leading to an increase in the number density of neutral donors magnetic-field-unbinding. The decrease in strength of the singlet transition, and ultimately of the triplet transition at high enough $B$, with a concomitant increase in the strength of the $\mathrm{D}^{0}$ transition is direct experimental evidence of this. Results for the relative strengths of on-well-center and off-well-center singlet lines are in very good agreement with recent calculations [18].

\subsection{Well-center donors as a probe of many-electron effects}

With increasing excess electron densities in the wells the two-electron $\mathrm{D}^{-}$-singlet and -triplet $\left(\mathrm{T}^{-}\right)$transitions evolve continuously into blue-shifted many-electron singlet-like and triplet-like bound magnetoplasmon transitions. The blue-shift of the collective excitations at integral $\nu$ results predominantly from exchange lowering of the many-body ground state [17]. The systematic behavior with excess electron density is seen clearly in Fig. 6a in which the differences between the positions of the transmission minima corresponding to the singlet-like transitions and those corresponding to CR are plotted vs. magnetic field for four $20 \mathrm{~nm}$ well-width samples all doped in the wells at $2 \times 10^{10} \mathrm{~cm}^{-2}$, but with barrier doping varying between $3.5 \times 10^{10} \mathrm{~cm}^{-2}$ and $2.8 \times 10^{11} \mathrm{~cm}^{-2}$. In addition to the large changes at integral $\nu$, anomalies are also observed near $\nu=1 / 3,2 / 3,4 / 3$, and perhaps $2 / 5$ as seen in Figs. 6a and b. Results for the normalized blue shifts of the singlet-like transitions relative to the $\mathrm{D}^{-}$-singlet line in the lowest barrier-doping-density sample (solid squares of Fig. 6a) are plotted in Fig. 6b. The values at integral $\nu$ are in reasonable agreement with recent many-body calculations [17]. In addition, for all samples studied the blue-shift of the many-electron singlet-like transition decreases with increasing magnetic field for $\nu<1$, approaching from above the transition energy of the two-electron $D^{-}$-singlet, even though there are as many as 15 electrons/donor. Concomitantly, the many-electron triplet-like transition loses strength. The qualitative behavior can be seen from Fig. 6a, but the systematic behavior is much more clearly evident in the normalized plot of Fig. $6 \mathrm{~b}$. Note that the normalized values for two different samples at $\nu=1$ are equal, and for $\nu<1$, 

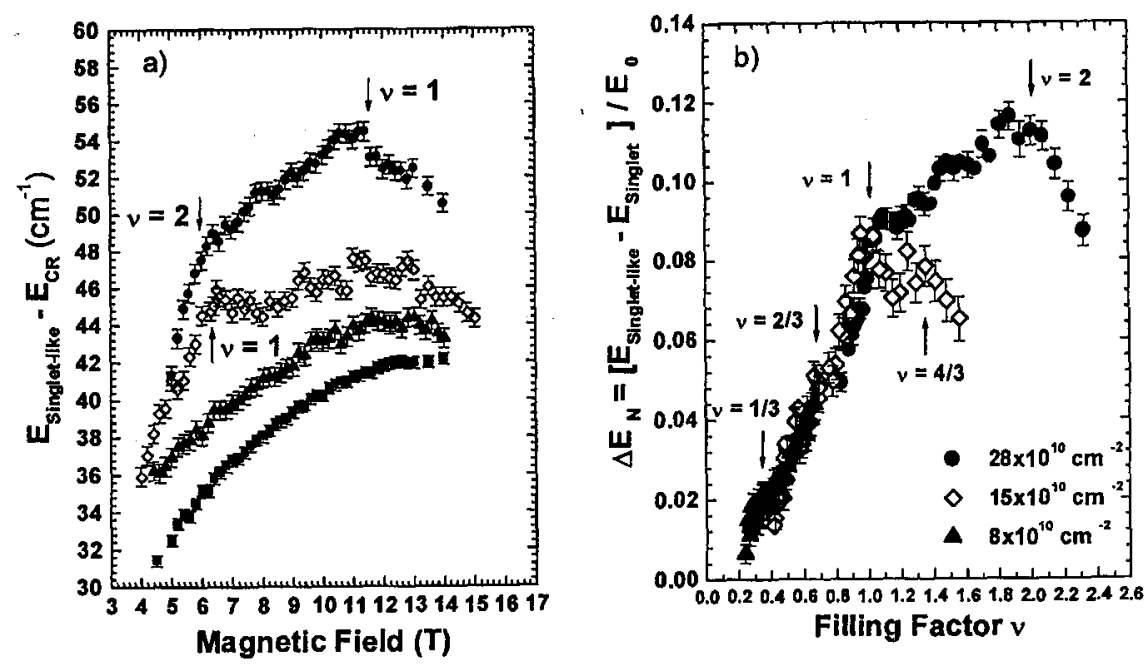

Fig. 6. (a) Differences between transition energies and $C R$ positions as a function of magnetic field for the $\mathrm{D}^{-}$-singlet transition ( () of a reference sample (barrier doping $3.5 \times 10^{10} \mathrm{~cm}^{-2}$ ), and the singlet-like transitions for barrier doping of $8 \times 10^{10} \mathrm{~cm}^{-2}$ (full triangle), $1.5 \times 10^{11} \mathrm{~cm}^{-2}(\diamond)$, and $2.8 \times 10^{11} \mathrm{~cm}^{-2}(\bullet)$. All MQW samples are structurally identical with 20 repetitions of $20 \mathrm{~nm}$ wells and $60 \mathrm{~nm}$ barriers. (b) Plots of the normalized blue-shifts as a function of filling factor. The normalizing energy is the $B$-dependent $2 \mathrm{D}$ neutral donor binding energy in the high field limit, $E_{0}=(\pi / 2)^{1 / 2}\left(e^{2} / \varepsilon \ell\right)$, with $\varepsilon$ - the background dielectric constant, and $\ell-$ the cyclotron orbit radius. Note: $\Delta E_{N}(\nu=2): \Delta E_{N}(\nu=1)=1.3: 1$. In the 2D, high-field limit the predicted ratio is $2: 1$.

the normalized data for all samples collapse onto a single curve, showing scaling behavior with $\nu$, and the many-body nature of the effects.

The systematic decrease in the blue shift for $\nu<1$ can be understood from exact diagonalization calculations for a system of nine electrons in the presence of a weak parabolic confining potential with a positive donor ion at the origin [14]. Results show that two electrons are always bound in a spin-singlet configuration near the positive charge, and the remaining electrons tend to move to larger (outer) orbits within the parabolic confining potential as they become available at higher magnetic fields. Figure 7 a shows the probability densities projected onto the single-particle Landau states $(N=0)$ characterized by orbit-center quantum number $O$ for the case $\nu>1$. This corresponds to a field such that eight Landau orbitals lie inside the well; orbitals 0 through 7 are occupied by spin-up electrons, with the $O=0$ orbital at the origin occupied by a spin-down electron. The charge distribution of spin-up polarized electrons is uniform in this case, and there is a large blue shift of the singlet-like transition. Figure $7 \mathrm{~b}$ shows the situation for $\nu=1$. In this higher field case, nine Landau orbitals lie inside the confining potential. But rather than the spin-polarized state that would obtain for noninteracting electrons with no positive charge, a partial hole is created in the $O=1$ orbital state for spin-up, and "most of" a singlet-electron pair resides at the origin. This is anal- 


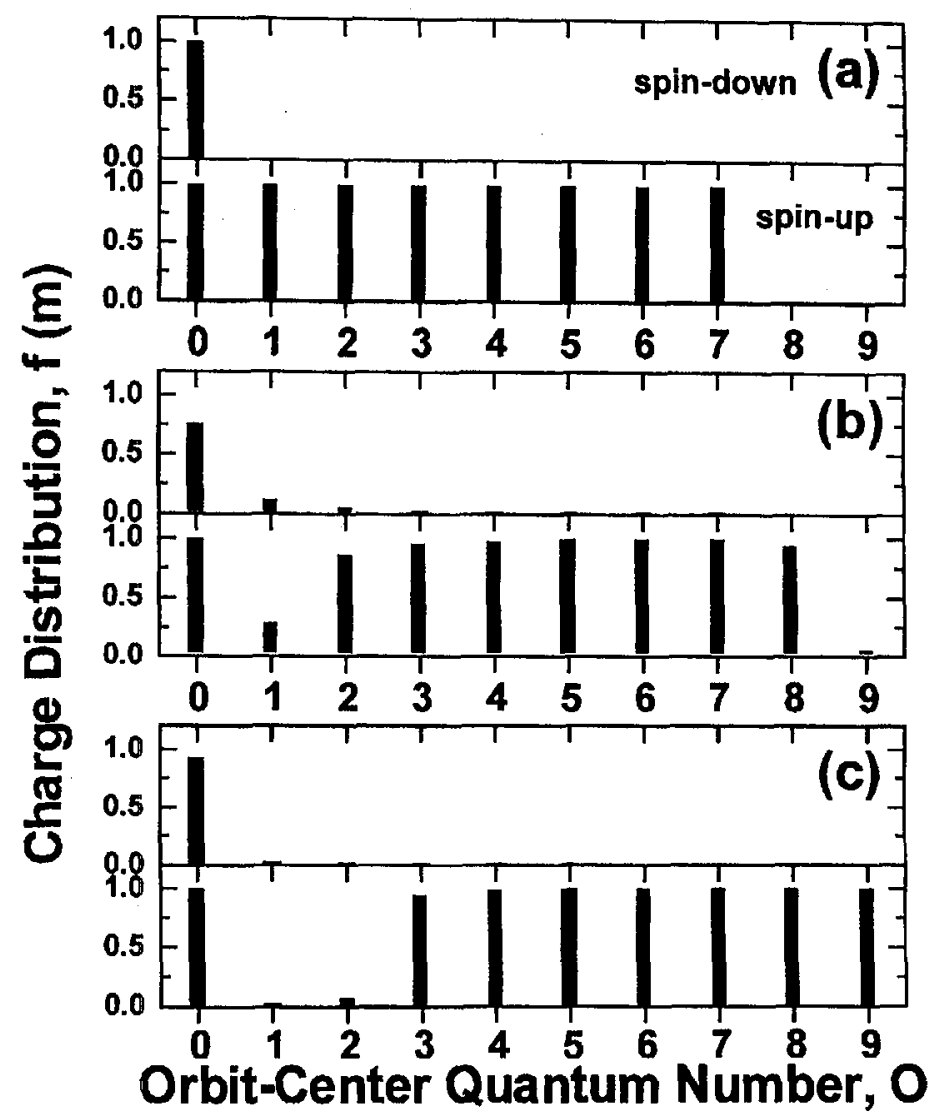

Fig. 7. The calculated electron charge distribution as a function of $O$ for the lowest $N=0 \mathrm{LL}$ for the model described in the text; (a) $\nu>1$, (b) $\nu=1$, and (c) $\nu<1$. The upper and lower panels in each case are for spin-down and spin-up electrons, respectively, and the unit on the vertical axis is the electron charge.

ogous to the spin-wave state predicted earlier by Hawrylak (Fig. 2c) [17]. In this case the triplet transition is weakened (since it requires population of the $O=1$ orbital), but there is still a large blue shift. For $\nu<1$ (Fig. 6c) a spin-singlet pair of electrons is localized at the positive charge $(O=0)$, there are holes in the $O=1$ and 2 states, and the outermost orbital inside the confining potential is fully occupied. The spin-singlet at the origin gradually becomes isolated from the remaining electrons, with a corresponding reduction in the blue-shift of the singlet-like transition. Excess electrons in the potential minimum are repelled from the negatively charged spin-singlet pair at the origin, and they also maintain the maximum possible separation from one another by populating the edge states to decrease the electron-electron repulsion and minimize the total energy. Thus at high fields a positive charge binding two spin-singlet electrons becomes spatially isolated from the remaining electrons with increasing field for $\nu<1$, and the 
optical properties of the many-electron system approach those of a two-electron $\mathrm{D}^{-}$-singlet configuration, as observed experimentally.

This model is justified physically by the existence of a lateral, long-range, fluctuating potential due to variations in the barrier doping density. When the characteristic magnetic length, $\ell$, becomes significantly smaller than the correlation length of the fluctuating potential, electrons tend to localize in the lowest potential minima of the fluctuating potential landscape. Statistically, a significant number of these potential minima are expected to be located laterally close to a positive donor ion in the well.

\subsection{Hydrostatic pressure tuning of many-electron effects}

Applied hydrostatic pressure can be used to vary the excess free electron density in modulation doped MQW structures [20]. Increasing pressure causes the $\Gamma$-point conduction-band edge (CBE) in the GaAs wells to cross the $X$-point CBE in the AlGaAs barriers [21], transferring electrons back to their parent donors in the barriers. This approach has the great advantage of permitting studies of many-electron effects as a function of electron density in a single MQW sample.

Hydrostatic pressure has been "married" recently to far infrared magnetospectroscopy. Initial experiments with a unique diamond anvil pressure cell have demonstrated the efficacy of the technique and permitted the evolution of the spectra to be explored as a function of excess electron density in a single MQW sample [22, 23]. Figure 8 shows some results from a systematic FTIR study of pressure effects on the same samples discussed in the previous section. Parts (a) and (b) compare results at two pressures, $28 \mathrm{kbar}$ (at the $\Gamma-X$ crossover) and $22 \mathrm{kbar}$ (below the $\Gamma-X$ crossover) on the sample doped at $3.5 \times 10^{10} \mathrm{~cm}^{-2}$ in the barriers. The effect of pressure, which decreases the electron density in the wells, is apparent. At $22 \mathrm{kbar}$ the spectrum is dominated by the $\mathrm{D}^{-}$-singlet line and free electron $\mathrm{CR}$, indicating that there are more than two electrons per donor ion in the wells. At $28 \mathrm{kbar}$ there is only a neutral donor $1 s-2 p^{+}$line, indicating that most of the electrons have been removed from the wells. Part (c) shows a spectrum for the sample with $2.8 \times 10^{11} \mathrm{~cm}^{-2}$ electrons per well at $22 \mathrm{kbar}$. From comparison with the lower density sample of part (b), a blue shift of about $11 \mathrm{~cm}^{-1}$ is obtained at this pressure, comparable to that observed at atmospheric pressure.

Extensive studies of this high-density sample at higher pressures (in $\approx 2 \mathrm{kbar}$ increments) show a systematic decrease in the blue shift with increasing pressure (decreasing excess electron density), in qualitative agreement with the atmospheric pressure studies on several samples described above. However, the magnetic field dependence of the blue shift is significantly stronger in these high-pressure studies than was found at atmospheric pressure. In the region of the $\Gamma$ (well)-X(barrier) crossover, the blue shift is larger, and it decreases strongly with field, approaching the isolated $\mathrm{D}^{-}$-singlet line more rapidly. This behavior is not presently understood. 


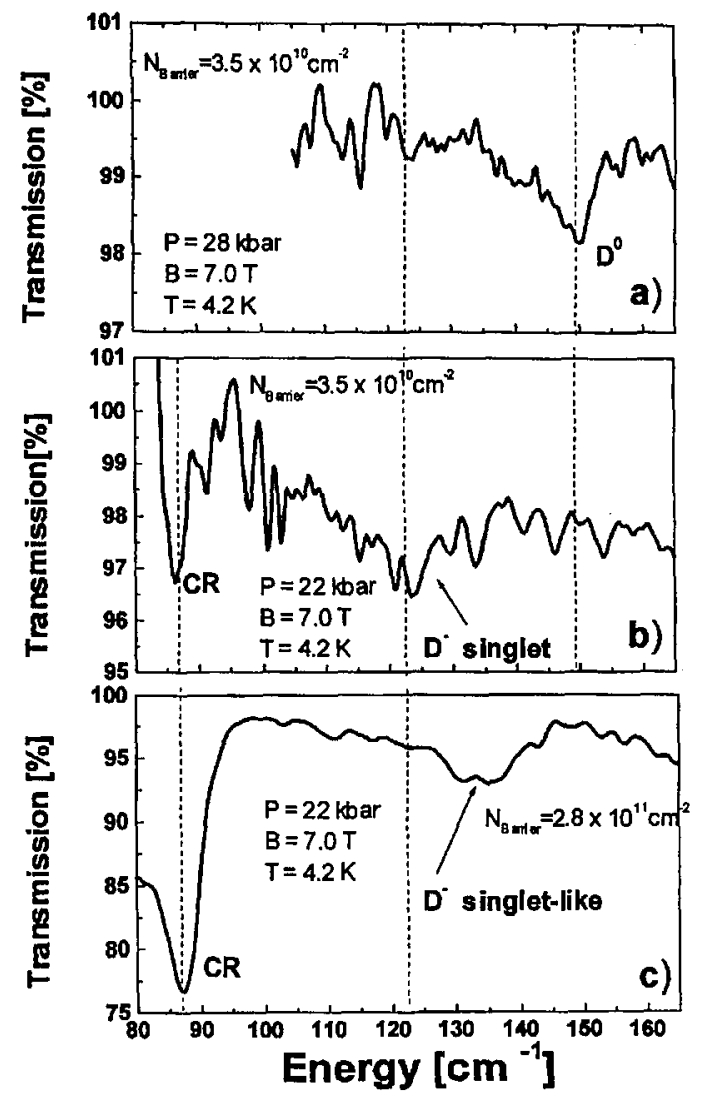

Fig. 8. Fourier transform magnetotransmission data at $7 \mathrm{~T}$ and $4.2 \mathrm{~K}$ : (a) data taken on a $20 \mathrm{~nm}$ well-width MQW sample (20 repetitions) $\delta$-doped in the well-centers at $2 \times 10^{10} \mathrm{~cm}^{-2}$ and in the $60 \mathrm{~nm}$ barrier-centers at $3.5 \times 10^{10} \mathrm{~cm}^{-2}$ at $28 \mathrm{kbar}$, (b) data taken at $22 \mathrm{kbar}$ on the same sample, and (c) data taken at $22 \mathrm{kbar}$ on an MQW (20 repetitrions) sample $\delta$-doped in the well-centers at $2 \times 10^{10} \mathrm{~cm}^{-2}$ and in the $60 \mathrm{~nm}$ barrier-centers at $2.8 \times 10^{11} \mathrm{~cm}^{-2}$.

\section{Acknowledgments}

The experimental work was supported in part by ONR (N00014-97-10858) and NSF (DMR-962409). B.D.M. is grateful to A.B. Dzyubenko and A.Yu. Sivachenko for helpful discussions, and for communication of unpublished results, and to R. Heron for a careful reading of the manuscript. The excellent samples were grown by Dr. W. Schaff.

\section{References}

[1] See e.g., B.D. McCombe, A. Petrou, in: Handbook on Semiconductors, Optical Properties of Semiconductors, Vol. 2, Ed. M. Balkanski, Elsevier Sci. Publ., Amsterdam 1994, p. 288; P.O. Holtz, A.C. Ferreira, Q.X. Zhao, B. Monemar, M. Sundaram, K. Campman, J.L. Merz, A.C. Gossard, in: Proc. 7th Int'l Conf. on Shallow-level Centers in Semiconductors, Eds. C.A.J. Ammerlaan, B. Pajot, World Scientific, Singapore 1996, p. 67 and references therein. 
[2] S. Huant, S.P. Najda, B. Etienne, Phys. Rev. Lett. 65, 1486 (1990).

[3] S. Holmes, J.-P. Cheng, B.D. McCombe, W. Schaff, Phys. Rev. Lett. 69, 2571 (1992); E.R. Muller, D.M. Larsen, J. Waldman, W.D. Goodhue, Phys. Rev. Lett. 68, 2204 (1992); A. Mandray, S. Huant, B. Etienne, Europhys. Lett. 20, 181 (1992).

[4] T. Pang, S.G. Louie, Phys. Rev. Lett. 65, 1635 (1990); D.M. Larsen, S.Y. McCann, Phys. Rev. B 45, 3485 (1991); D.M. Larsen, S.Y. McCann, Phys. Rev. B 46, 3966 (1992); A.H. MacDonald, Solid State Commun. 84, 109 (1992); A.B. Dzyubenko, A.Yu. Sivachenko, Phys. Rev. B 48, 14690 (1993).

[5] W.J. Li, J.L. Wang, B.D. McCombe, J.-P. Cheng, W. Schaff, Surf. Sci. 305, 215 (1994).

[6] A.B. Dzyubenko, A. Mandray, S. Huant, A.Yu. Sivachenko, B. Etienne, Phys. Rev. $B$ 50, 4687 (1994).

[7] S.R. Ryu, Z.X. Jiang, W.J. Li, B.D. McCombe, W. Schaff, Phys. Rev. B 54 (Rapid Commun.), R11086 (1996).

[8] Jia-Lin Zhu, Sheng Xu, J. Phys., Condens. Matter 6, L299 (1994).

[9] H.L. Fox, D.M. Larsen, Phys. Rev. B 51, 10709 (1995).

[10] I.K. Marmorkos, V.A. Schweigert, F.M. Peeters, Phys. Rev. B 55, 5065 (1997).

[11] Z. X. Jiang, B.D. McCombe, J.L. Zhu, W. Schaff, Phys. Rev. B 56, R1692 (1997).

[12] J.- P. Cheng, Y.J. Wang, B.D. McCombe, W. Schaff, Phys. Rev. Lett. 70, 489 (1993).

[13] Z.X. Jiang, S.R. Ryu, B.D. McCombe, Semicond. Sci. Technol. 11, 1608 (1996); Z.X. Jiang, B.D. McCombe, P. Hawrylak, in: Proc. 23rd Int'l Conf. on the Physics of Semiconductors, Berlin 1997, Eds. M. Scheffler, R. Zimmermann, Vol. 3, World Scientific, Singapore 1996, p. 2535.

[14] Z.X. Jiang, B.D. McCombe, P. Hawrylak, Phys. Rev. Lett. 81, 3499 (1998).

[15] See e.g., R. Greene, K.K. Bajaj, Phys. Rev. B 31, 913 (1985).

[16] J.-P. Cheng, B.D. McCombe, Phys. Rev. B 42, 7626 (1990).

[17] A.B. Dzyubenko, Yu.E. Lozovik, JETP 77, 617 (1993); P. Hawrylak, Phys. Rev. Lett. 72, 2943 (1994).

[18] A.B. Dzyubenko, A.Yu. Sivachenko, private communication and to be published.

[19] C. Riva, V.A. Schweigert, F.M. Peeters, Phys. Rev. B 57, 15392 (1998).

[20] See e.g., D.K. Maude, J.C. Portal, L. Dmowski, T. Foster, L. Eaves, M. Nathan, M. Heiblum, J.J. Harris, R.B. Beall, Phys. Rev. Lett. 59, 815 (1987).

[21] C.G. Van de Walle, Phys. Rev. B 39, 1871 (1989).

[22] Z.X. Jiang, R.J. Chen, J.G. Tischler, B.A. Weinstein, B.D. McCombe, Phys. Status Solidi $B$ 198, 41 (1996).

[23] J.G. Tischler, S.K. Singh, H.A. Nickel, G.S. Herold, Z.X. Jiang, B.D. McCombe, B.A. Weinstein, Phys. Status Solidi B 211, 131 (1998). 